\title{
The Field of View is More Useful in Golfers than Regular Exercisers
}

\author{
Karen Murphy ${ }^{1,2}$ \\ ${ }^{1}$ Menzies Health Institute Queensland, Australia \\ ${ }^{2}$ School of Applied Psychology, Griffith University, Australia
}

ABSTRACT

Superior visual attention skills are vital for excellent sports performance. This study used a cognitive skills approach to examine expert and novice differences in a visual spatial attention task. Thirtytwo males aged 18 to 42 years completed this study in return for course credit or monetary incentive. Participants were expert golfers $(N=18)$ or exercise controls $(N=14)$. Spatial attention was assessed using the useful field of view task which required participants to locate a target shown $10^{\circ}$, $20^{\circ}$, and $30^{\circ}$ of eccentricity from centre in very brief presentations. At each degree of eccentricity, golfers were more accurate at locating the target than the exercise controls. These results provide support for the broad transfer hypothesis by demonstrating a link between golf expertise and better performance on an objective measure of spatial attention skills. Therefore, it appears that sports expertise can transfer to expertise in non-sport related tasks.

\section{INTRODUCTION}

The link between high levels of sports performance and athletes' cognitive skills is of significant interest to athletes, coaches, sport scientists, and researchers. To understand the link between cognitive skills and sports performance, many studies have adopted the expert performance approach (Mann, Williams, Ward, \& Janelle, 2007; Starkes \& Ericsson, 2003), which investigates athletes' cognitive skills in sport contexts or sports related tasks (e.g., Voss, Kramer, Basak, Prakash, \& Roberts, 2010). For example, expert athletes have been found to have better visual attention capabilities or abilities to utilise the attended visual information than novice athletes or non-athletes in sport related tasks or contexts (e.g., Abernethy \& Russell, 1987; Beilock, Wierenag, \& Carr, 2002; Memmert, 2006; Savelsbergh, Williams, van Der Kemp, \& Ward, 2002). Expert athletes have also been shown to outperform novices on sport-specific measures of decision making, pattern recognition, spatial memory, and memory recall (e.g., Abernethy, Baker, \& Côté, 2005; Dijkstra, MacMahon, \& Misirlisoy, 2008; Starkes \& Ericsson, 2003; Williams \& Ford, 2008).

The results of these expert performance approach studies support the narrow transfer hypothesis (Simons \& Chabris, 2010, also referred to as the specific processing hypothesis Furley \& Memmert, 2011).
According to this hypothesis, people with years of extensive experience in sport, music, or chess only differ from the average person in skills that directly relate to their area of expertise. Hence, these superior skills would not be expected to translate into better performance outside the sporting context.

The broad transfer hypothesis (Furley \& Memmert, 2011) proposes that expert skills acquired in one domain can transfer to better performance in other, remotely related domains. For example, expertise or training on action video games can result in better performance on standard measures of visual attention (Green \& Bavelier, 2003, 2006). There is also research with expert athletes that supports the broad transfer hypothesis. This research uses the cognitive component skills approach (Furley \& Memmert, 2010; Voss et al., 2010) and compares experienced athletes with nonathletes or less experienced athletes on domain general measures of cognitive abilities. Studies have shown that generic attention tasks produced smaller attentional benefits and costs

Corresponding author: Karen Murphy, School of Applied Psychology, Griffith University, Gold Coast, Queensland 4222, Australia. Phone: +617-5678 8952. E-mail: k.murphy@griffith.edu.au 
for athletes from open skill sports compared to controls (e.g., Nougier, Ripoll, \& Stein, 1989; Nougier \& Rossi, 1999; Nougier, Stein, \& Bonnel, 1991) and that professional volleyball players outperformed novices on a computerised cued target detection task (Castiello \& Umiltà, 1992). Thus, consistent with the broad transfer hypothesis (Furley \& Memmert, 2011), an athlete's superior attention skills are not limited to the sports domain but are evident in standard attention tasks.

However, not all research using the cognitive skills approach supports the broad transfer hypothesis. The visuospatial capacity of expert basketball players and nonathlete college students was examined using the Corsi block-tapping task (Furley \& Memmert, 2010). Contrary to expectation, there was no task performance difference between these two groups. However, Allen, Fioratou, and McGeorge (2011) noted that the Corsi block-tapping task may not have been sufficiently sensitive to detect performance advantages for the experts, or the nonathlete control group may have had good visuospatial skills due to video game playing. Thus, either or both of these suggestions could account for the lack of group differences on the Corsi block-tapping task in Furley and Memmert's (2010) study, rather than the results being consistent with the narrow transfer hypothesis.

Using a cognitive components skills approach, Memmert, Simons, and Grimme (2009) examined attentional differences between team handball experts, expert athletes from non-team sports, and a combined group of novice athletes (with no more than two years of formal experience in any sport) and nonathletes. The study used the multiple object tracking task (track multiple moving targets among distractors; Pylyshyn \& Storm, 1988; Trick, Jaspers-Fayer, \& Sethi, 2005) and an inattentional blindness task (detect unexpected objects within participants' central field of view; Mack \& Rock, 1998; Most, Scholl, Clifford, \& Simons, 2005). The study also used the useful field of view (UFOV) task to assess the total area of the visual field which allows individuals to detect relevant information without eye or head movement (Ball, Beard, Roenker, Miller, \& Griggs, 1988). As the targets are presented briefly at varying degrees of visual angle from the centre of the visual field, the UFOV provides an estimate of the spatial distribution of attention (Feng, Spence, \& Pratt, 2007; Green \& Bavelier, 2003). Memmert et al. did not find performance differences between the groups on any of these three tasks.

While these results might suggest that basic visual attention skills are not associated with sport expertise and, therefore, not consistent with the broad transfer hypothesis, there are several reasons why group differences may not have been evident in this study. Memmert et al. (2009) included novice athletes and nonathletes within one group of participants. Thus, although the novice athletes were not experts, it is possible that their visual attention skills may have been closer to those of the expert athletes, thereby masking any group differences. Further, there was no measure of video game player status in the study, which is critical given video game playing has been linked to better UFOV performance (Feng et al., 2007; Green \& Bavelier, 2003, 2006; although see Murphy \& Spencer, 2009, for a different result). Consequently, if there were a reasonable number of gamers in the nonathlete and novice athlete group (Allen et al., 2011), their video games skills may have allowed them to perform the UFOV task at the same level as the expert athletes. Additionally, regular aerobic exercise has been shown to enhance cognitive function and alter structural and functional aspects of the brain (e.g., Kramer \& Erickson, 2007a, 2007b). Therefore, aerobically fit people may perform better on the visual attention tasks than unfit participants. By including novice athletes within the control group, this may have minimised the potential for Memmert et al. to find group differences as their fitness levels may have allowed them to perform similarly to the experts on the attention tasks.

The current study used a cognitive component skills approach (Furley \& Memmert, 2010; Voss et al., 2010) to compare a group of expert golfers against a control group of regular exercisers on a modified UFOV task (based on the task used by Green \& Bavelier, 2003). Prior studies have shown that action video game players have a more effective UFOV compared to non-gamers (e.g., Feng et al., 2007; Green \& Bavelier, 2003, 2006), which is consistent with the broad transfer hypothesis (Furley \& Memmert, 2011). The current study extended work in this area by using the UFOV task as an objective measure of spatial attention skill in expert golfers and compared their performance on this task to a control group. Golfers were selected as the expert participants for this study, as they use a variety of different attentional skills during training and competition (see the analysis by Bernier, Codron, Thienot, \& Fournier, 2011). This suggests that golfers have the potential for excellent visual attention skills, including spatial attention skills, an aspect of attention that has not been examined in golfers to date. Most visual attention research on golfers has used the sport-specific approach (relevant to the narrow transfer hypothesis) and, therefore, little is known about the applicability of golf expertise in relation to the broad transfer hypothesis (Furley \& Memmert, 2011) or golfers' performance on an objective measure of spatial attention skills. The current study addressed this issue.

Given the cognitive benefits of aerobic exercise (Kramer \& Erickson, 2007a, 2007b) and the impact this may have had on the results of Memmert et al. (2009), this study employed a physically fit age-matched control group (Voss et al., 2010), to minimise the impact of this variable on the UFOV performance. Finally, as noted above in relation to the study by Memmert et al. and following the suggestions of Furley and Memmert (2011), this study also assessed time spent playing video games to control for cognitive adaption due to this activity.

Based on the literature reviewed above, it is proposed that if the golfers and exercise controls do not differ on the UFOV task, then this would support the narrow transfer hypothesis (Furley \& Memmert, 2011) and suggest that only domain-specific attention skills are enhanced by sports expertise. However, if the golfers show superior UFOV performance compared to the exercise control group, this would support the broad transfer hypothesis (Furley \& Memmert, 2011). Additionally, this study sought to examine if there was an association between golf skill level (handicap) and UFOV task performance. 


\section{METHOD}

\section{Participants}

Thirty-two males aged 18 to 42 years $\left(M_{\text {age }}=24.02, S D=6.21\right)$ voluntarily completed this study in return for course credit or monetary reimbursement. Only male participants completed the experiment, as previous studies have shown gender differences in the performance of the UFOV task (e.g., Feng et al., 2007; Terlecki \& Newcombe, 2005; Voyer, Voyer, \& Bryden, 1995). All participants had normal colour vision and normal or corrected to normal visual acuity. Participants were experienced competitive golfers enrolled in the Griffith University Professional Golf Association program $(N=18)$ or exercise control participants recruited from the Griffith University student population $(N=14)$. The golfers had played competition for 4 to 17 years $(M=$ $7.61, S D=3.09)$, with handicaps ranging from 3 to $13(M=6.5, S D=$ $2.77)$. The exercise control participants engaged in a regular aerobic exercise program (swimming, running, aerobic gym sessions) but did not participate in competitive sport. The golfers and controls were matched on age, $t(30)=-.187, p=.853$, and hours per week spent playing video games, $t(30)=-.911, p=.369$. Table 1 presents the relevant descriptive statistics for the between-group comparisons presented here. There was no difference between the golfers and controls in the number of times per week they exercised or trained, $t(30)=-.661, p=.51$. The training sessions for the golfers were of longer duration than those of the controls, $t(30)=5.46, p<.001$, and overall, the golfers spent more hours per week training and exercising than the controls, $t(30)=-3.83$, $p=.001$.

\section{Materials}

The UFOV task was run on a Pentium IV 2.66GHz PC with a Dell Ultra flat CRT monitor using the DMDX program (Forster \& Forster, 2003), which recorded the accuracy of participant responses for each trial. Each trial began with a central fixation square $\left(4^{\circ} \times 4^{\circ}\right)$ presented for $100 \mathrm{~ms}$, followed by a set of 24 small white squares $\left(4^{\circ} \times 4^{\circ}\right)$ that formed a circular wheel with eight spokes. On each trial, the target (a filled triangle within a $3^{\circ} \times 3^{\circ}$ circle) appeared on one of the eight spokes at one of three possible eccentricities from the centre. The targets were presented at $10^{\circ}, 20^{\circ}$, or $30^{\circ}$ from the centre of the visual field, based on the UFOV task specifications used in previous research (e.g., Ball et al., 1988; Feng et al., 2007; Green \& Bavelier, 2003). There were 24 possible locations at which the target could appear. The target appeared for 15 $\mathrm{ms}$ at $10^{\circ}$ and $30 \mathrm{~ms}$ at $20^{\circ}$ or $30^{\circ}$. The difference in target presentation times was intended to control for the increased difficulty of detecting the target at $20^{\circ}$ or $30^{\circ}$ as opposed to at $10^{\circ}$ (Green \& Bavelier, 2003). A mask screen (i.e., a coloured pattern) appeared for $200 \mathrm{~ms}$ following the target, and then the response screen was shown, displaying eight intersecting lines in the form of the wheel. Participants indicated the spoke on which the target appeared using the numbers 1 to 8 on the number pad to match the spoke labels presented. Figure 1 presents the sequence of stimuli shown within each UFOV trial.

\section{TABLE 1.}

Means and Standard Deviations for the Participant Characteristics for the Golfers $(N=18)$ and the Exercise Control $(N=14)$ Group

\begin{tabular}{|c|c|c|c|c|}
\hline \multirow[b]{2}{*}{ Measure } & \multicolumn{2}{|c|}{ Golf group } & \multicolumn{2}{|c|}{ Exercise control group } \\
\hline & $M$ & $S D$ & $M$ & $S D$ \\
\hline Age in years & 24.61 & 6.99 & 24.14 & 7.03 \\
\hline Number weekly training/exercise sessions & 4.17 & 1.55 & 3.79 & 1.70 \\
\hline Duration weekly training/exercise sessions (hrs) & 3.28 & 1.33 & 1.23 & 0.47 \\
\hline Hours per week training or exercising & 13.26 & 8.09 & 4.70 & 2.29 \\
\hline Hours per week playing video games & 2.06 & 3.42 & 1.11 & 2.19 \\
\hline
\end{tabular}

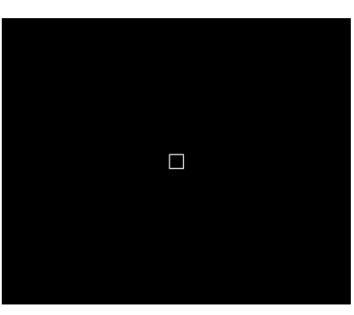

Fixation point

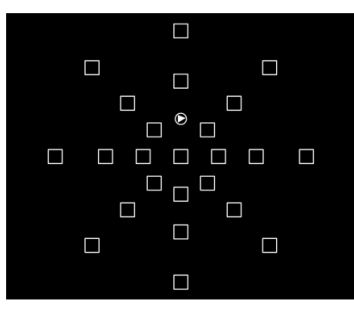

Target Display

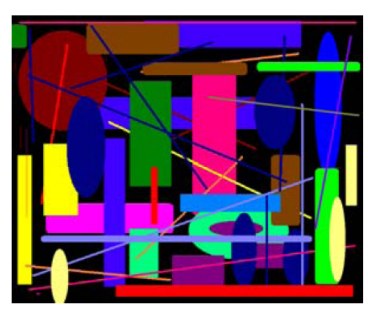

Patterned Mask

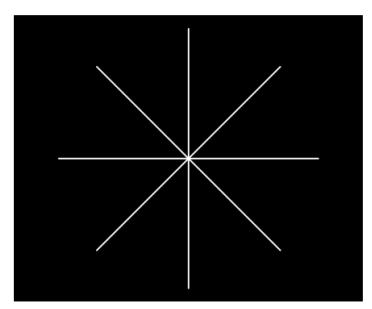

Response Screen ( 1 to 8 on monitor frame)

FIGURE 1.

The trial sequence presented (left to right) for the Useful Field of View task showing the target located at $10^{\circ}$ of eccentricity from centre. 


\section{Procedure}

Participants provided written informed consent, and they completed a brief questionnaire eliciting demographic information, sport and exercise history, and video game playing history. This was followed by UFOV task, where participants were seated $30 \mathrm{~cm}$ from the computer screen and asked to locate the position of the target on each trial. Participants completed 20 practice trials followed by 240 randomly presented experimental trials, 80 trials at each target eccentricity.

\section{Design and Analysis}

The independent variables were Group (2), a between-subjects factor and Target Eccentricity (3), a within-subject factor. The dependent variable was Accuracy of Target Location (\% correct) in the UFOV task.

Although the groups did not differ on hours of video game playing per week, as the research has shown that video game experience affects performance on the UFOV task (Feng et al., 2007; Green \& Bavelier, $2003,2006)$, this measure was used as a covariate in the analysis. As the participants' ages ranged from 18 to 42 years and age has been shown to differentially affect UFOV task performance in young and middle aged adults (Ball et al., 1988), it was also used as a covariate in this study. Physical fitness training has been shown to enhance cognitive functioning and alter structural and functional aspects of the brain (Kramer \& Erickson, 2007a, 2007b). The number of hours per week spent training or exercising was another covariate in the UFOV analysis, as the groups differed on this measure.

The UFOV accuracy data were analyzed using a $2 \times 3$ (Group [golfers, exercise controls $] \times$ Target Location $\left[10^{\circ}, 20^{\circ}, 30^{\circ}\right.$ from the centre $\left.]\right)$ mixed factorial analysis of covariance (ANCOVA) with age and hours per week spent playing video games and exercising as the covariates.

\section{RESULTS}

The data analysis revealed that after controlling for age, hours exercising and playing video games, there was a main effect of target eccentricity on accuracy, $F(2,54)=4.59, p=.014, \eta_{\mathrm{p}}{ }^{2}=.15$. Post hoc pairwise comparisons showed that accuracy was higher when the targets were located at $10^{\circ}$ of visual angle from centre $(M=84.32 \%, S E=2.65)$ than when targets were located at $20^{\circ}$ of visual angle from centre $(M=$ $78.48 \%, S E=1.72$ ). Target detection at both $10^{\circ}$ and $20^{\circ}$ of eccentricity was more accurate than when the targets were located at $30^{\circ}$ of visual angle from the centre $(M=54.42 \%, S E=2.51)$, all $p$ s $\leq .001$.

Overall target detection accuracy was higher for the golfers $(M=$ $79.40 \%, S E=2.63)$ than the controls $(M=65.41 \%, S E=3.06)$ after controlling for age, hours spent exercising and playing video games, $F(1,27)=10.03, p=.004, \eta_{\mathrm{p}}{ }^{2}=.27$. The interaction between Group and Target Eccentricity was not significant, $F(2,54)=2.00, p=.15, \eta_{\mathrm{p}}{ }^{2}=.07$, indicating that the group difference was consistent at each degree of target eccentricity. This was confirmed by running post hoc contrasts comparing the accuracy of the golfers and controls at each degree of target eccentricity. Compared to controls, the golfers had higher detection accuracies for targets located at $10^{\circ}, F(1,27)=3.11, p=.08, \eta_{\mathrm{p}}{ }^{2}=$
TABLE 2.

Useful field of view (UOFV) Task Percentage Mean Accuracy $(S E)$ for the Golfers $(N=18)$ and the Exercise Controls $(N=14)$

\begin{tabular}{ccccc}
\hline & \multicolumn{2}{c}{ Golfers } & \multicolumn{2}{c}{ Exercise control } \\
$\begin{array}{c}\text { Target eccentricity } \\
\text { (degrees) }\end{array}$ & $M$ & $S E$ & $M$ & $S E$ \\
\hline 10 & 89.87 & 3.75 & 78.77 & 4.37 \\
20 & 83.47 & 2.50 & 73.49 & 2.90 \\
30 & 64.86 & 3.65 & 43.97 & 4.23 \\
\hline
\end{tabular}

.10 (marginal trend towards significance), at $20^{\circ}, F(1,27)=5.68, p=$ $.024, \eta_{\mathrm{p}}{ }^{2}=.17$, and at $30^{\circ} F(1,27)=11.66, p=.002, \eta_{\mathrm{p}}{ }^{2}=.30$, of visual angle from centre. See Table 2 for the relevant descriptive statistics.

To investigate if golf skill level or expertise was related to performance on the UFOV task, a correlation analysis examined the association between the golfers' handicaps and accuracy at each degree of target displacement from centre. At all three degrees of eccentricity, there was no significant correlation between target accuracy and golf handicap $\left(10^{\circ}, r=-.23, p=.361 ; 20^{\circ}, r=-.28, p=.258 ; 30^{\circ}, r=-.12, p=.628\right)$.

\section{DISCUSSION}

Consistent with prior research and the standard UFOV effect (Ball et al., 1988; Murphy \& Spencer, 2009), participants showed a decrement in target detection accuracy with increasing eccentricity. The superior target detection performance at all eccentricities for the golfers (marginally significant trend at $10^{\circ}$ eccentricity) compared to the controls is consistent with the differences reported for video game players and non-video game players on a UFOV task (Feng et al., 2007; Green \& Bavelier, 2003, 2006). The accuracy levels for the golfers and the exercise controls in this study were comparable to the video game players' and non-video game players' performances reported by Green and Bavelier (2003). Consequently, the superior UFOV performance for the golfers compared to controls in this study cannot be explained by uncharacteristically poor UFOV performance in the control group.

The results of the current study do not support the narrow transfer hypothesis (Simons \& Chabris, 2010). Instead, the better performance of the golfers on the UFOV task is consistent with the broad transfer hypothesis (Furley \& Memmert, 2011) which proposes that skills acquired in one domain (enhanced spatial attention through golf) can be transferred to better skills in other arenas (better performance on a laboratory task that measures spatial attention). There were no significant correlations between golf handicap and UFOV performance at the three degrees of target eccentricity. These nonsignificant results were most likely due to the small sample size for the golfers $(N=18)$ and do not detract from the group differences evident on the UFOV task. Thus, to the author's knowledge, this is the first study to demonstrate a link between golf expertise and enhanced spatial attention skills in a nonsporting context. 
However, it should be noted that the cross-sectional design of this study does not allow for the determination of a causal link between golf expertise and enhanced visual spatial attention skills. Future studies could track the developmental trajectory of visual attention skills for golfers across their career to assess attentional improvements across time. This would provide evidence of a causal link between good attentional skills and golf skill level.

The results of the current study differ from Memmert et al. (2009), who did not find any UFOV performance differences between expert handballers, expert non-team sports athletes, and a combined group of a novice athletes and nonathletes. However, there are several possible explanations for this. Firstly, Memmert et al. included both novice athletes and nonathletes in their control group while controls were regular aerobic exercisers and not competitive athletes in the current study. As noted previously, including novice athletes within Memmert et al.s control group may have masked any group differences as the novices' visual attention skills may be similar to that of the expert athletes. Secondly, unlike the current study, video game expertise was not measured or controlled for in Memmert et al.s study. It is therefore possible that at least some of their control participants were expert video game players (i.e., played at least 4 hours per week of action video games as defined by Green and Bavelier, 2003). Consequently, these control video game players may have had excellent visuospatial skills and, therefore, performed as well as the expert athletes on the UFOV task. Thirdly, Memmert et al's study did not control for their participants' aerobic activity levels. Inclusion of novice athletes within the control group may have resulted in this group having reasonable levels of aerobic fitness, which may have masked any group differences. The current study addressed this issue by using a measure of physical activity as a covariate.

Given that golf is a self-paced target sport, it could be argued that golfers should not show superior spatial attention skills compared to the exercise controls. However, research with athletes from other sports and golfers may assist with understanding the outcome of this study. Recently, Hüttermann, Memmert, and Simons (2014) demonstrated that the size and shape of athletes' attentional spotlights varied with their expertise for specific types of sports. Consequently, golfers' attention skills may be linked with the attentional skills they use in training and competition. Bernier et al's (2011) qualitative study of golfers' attentional foci in training and competition revealed that golfers focused on process (e.g., aspects of technique), result (e.g., movement outcomes, ball landing location), psychological state (e.g., motivation and emotion), and the environment (e.g., task-related elements such as wind or distance and irrelevant elements like birds or helicopters). The analysis also revealed that golfers focused on sense (kinaesthetic, visual, and auditory), reality (e.g., angle of the slopes before a shot or imagining the ball roll), and deliberate control of attention (what to focus on, e.g., rhythm). Bernier at al. also reported the sequences of attentional foci across time, which included things such as attending to the distance, wind, shadow, and shot rhythm. Therefore, golfers do not have a static attentional focus during their game. Instead, golfers move between a broad and narrow attentional focus and vary their atten- tional focus across time. Consequently, golf demands good attentional flexibility and these athletes employ a range of attention skills, including spatial attention, to focus on relevant information efficiently.

The current research provides support for the broad transfer hypothesis (Furley \& Memmert, 2011) by demonstrating that golfers had superior UFOV performance compared to a group of aerobically fit control participants. These results provide a significant contribution to the literature by showing a link between golf expertise and enhanced spatial attention skills, thereby supporting the notion that expertise in one domain can transfer to expertise in another unrelated domain. However, due to the cross-sectional design of this study further research is needed to establish a causal link between these factors. Future studies should also examine differences between athletes from various sports (experts and novices) and exercise controls on a range of standard measures of cognitive performance. This would allow examination of the range of cognitive processes (e.g., attention, memory, inhibition) that may be linked to sports expertise and provide a significant contribution to research in this area using the cognitive skills approach. The identification of a series of standard tasks that assess relevant aspects of cognitive function associated with certain sports could then be used to assist with talent identification schemes.

\section{AUTHOR NOTE}

Funding for this study was provided by a Griffith University Research Grant awarded to Karen Murphy. Thanks to Joan Caelli and Linda Peach for their assistance with the data collection in this study.

\section{REFERENCES}

Abernethy, B., Baker, J., \& Côté, J. (2005). Transfer of pattern recall skills may contribute to the development of sport expertise. Applied Cognitive Psychology, 19, 705-718. doi: 10.1002/ acp. 1102

Abernethy, B., \& Russell, D. G. (1987). The relationship between expertise and visual search strategy in a racquet sport. Human Movement Science, 6, 283-319. doi: 10.1016/0167-9457(87)90001-7

Allen, R., Fioratou, E., \& McGeorge, P. (2011). Cognitive adaptation: Spatial memory or attentional processing. A comment on Furley and Memmert (2010). Perceptual and Motor Skills, 112, 243-246. doi: 10.2466/04.22.PMS.112.1.243-246 |WWW

Ball, K. K., Beard, B. L., Roenker, D. L., Miller, R. L., \& Griggs, D. S. (1988). Age and visual search: Expanding the useful field of view. Journal of the Optical Society of America A, 5, 2210-2219. doi: 10.1364/JOSAA.5.002210 www

Beilock, S. L., Wierenga, S. A., \& Carr, T. H. (2002). Expertise, attention and memory in sensorimotor skill execution: Impact of novel task constraints on dual-task performance and episodic memory. Quarterly Journal of Experimental Psychology, 55A, 1211-1240. doi: 10.1080/02724980244000170 WwW

Bernier, M., Codron, R., Thienot, E., \& Fournier, J. F. (2011). The attentional focus of expert golfers in training and competition: $A$ naturalistic investigation. Journal of Applied Sport Psychology, 23, 326-341. doi: 10.1080/10413200.2011.561518 
Castiello, U., \& Umiltà, C. (1992). Orienting of attention in volleyball players. International Journal of Sport Psychology, 23, 301-301.

Dijkstra, K., MacMahon, C., \& Misirlisoy, M. (2008). The effects of golf expertise and presentation modality on memory for golf and everyday items. Acta Psychologica, 128, 298-303. doi: 10.1016/j.actpsy.2008.03.001 [WWW]

Feng, J., Spence, I., \& Pratt, J. (2007). Playing an action video game reduces gender differences in spatial cognition. Psychological Science, 18, 850-855. doi: 10.1111/j.1467-9280.2007.01990.x WWW

Forster, K. I., \& Forster, J. C. (2003). DMDX: A windows display program with millisecond accuracy. Behaviour Research Methods, Instruments, and Computers, 35, 116-124. doi: 10.3758/ BF03195503 WWW

Furley, P., \& Memmert, D. (2010). Differences in spatial working memory as a function of team sports expertise: The Corsi blocktapping task in sport psychological assessment. Perceptual and Motor Skills, 110, 801-808. doi: 10.2466/pms.110.3.801-808 WWW

Furley, P., \& Memmert, D. (2011). Studying cognitive adaptations in the field of sport: Broad or narrow transfer? A comment on Allen, Fioratou, and McGeorge (2011). Perceptual and Motor Skills, 113, 481-488. doi: 10.2466/05.23.PMS.113.5.481-488 WWW

Green, C. S., \& Bavelier, D. (2003). Action video game modifies visual selective attention. Nature, 423, 534-537. doi: 10.1038/

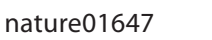

Green, C. S., \& Bavelier, D. (2006). Effect of action video games on the spatial distribution of visuospatial attention. Journal of Experimental Psychology: Human Perception and Performance, 32, 1465-1478. doi: 10.1037/0096-1523.32.6.1465 wwW

Hüttermann, S., Memmert, D., \& Simons, D. J. (2014). The size and shape of the attentional "spotlight" varies with differences in sports expertise. Journal of Experimental Psychology: Applied, 20, 147-157. doi: 10.1037/xap0000012 |WWw

Kramer, A. F., \& Erickson, K. I. (2007a). Capitalizing on cortical plasticity: Influence of physical activity on cognition and brain function. Trends in Cognitive Sciences, 11, 342-348. doi: 10.1016/j.tics.2007.06.009 wWW

Kramer, A. F., \& Erickson, K. I. (2007b). Effects of physical activity on cognition, well-being, and brain: Human interventions. Alzheimer's and Dementia, 3, S45-S51. doi: 10.1016/j. jalz.2007.01.008 www

Mack, A., \& Rock, I. (1998). Inattentional blindness. Cambridge, MA: MIT Press.

Mann, D. T., Williams, A. M., Ward, P., \& Janelle, C. M. (2007). Perceptual-cognitive expertise in sport: A meta-analysis. Journal of Sport and Exercise Psychology, 29, 457-478. doi: 10.1123/jsep.29.4.457 (www

Memmert, D. (2006). The effects of eye movements, age, and expertise on inattentional blindness. Consciousness and Cognition, 15, 620-627. doi: 10.1016/j.concog.2006.01.001
Memmert, D., Simons, D. J., \& Grimme, T. (2009). The relationship between visual attention and expertise in sports. Psychology of Sport and Exercise, 10, 146-151. doi: 10.1016/j. psychsport.2008.06.002

Most, S. B., Scholl, B. J., Clifford, E. R., \& Simons, D. J. (2005). What you see is what you set: Sustained inattentional blindness and the capture of awareness. Psychological Review, 112, 217-242. doi: 10.1037/0033-295X.112.1.217 |wWw

Murphy, K., \& Spencer, A. (2009). Playing video games does not make for better visual attention skills. Journal of Articles in Support of the Null Hypothesis, 6, 1-20.

Nougier, V., Ripoll, H., \& Stein, J. F. (1989). Orienting of attention with highly skilled athletes. International Journal of Sport Psychology, 20, 205-223.

Nougier, V., \& Rossi, B. (1999). The development of expertise in the orienteering of attention. International Journal of Sport Psychology, 30, 246-260.

Nougier, V., Stein, J. F., \& Bonnel, A. M. (1991). Information processing in sport and orienting of attention. International Journal of Sport Psychology, 22, 307-327.

Pylyshyn, Z. W., \& Storm, R. W. (1988). Tracking multiple independent targets: Evidence for a parallel tracking mechanism. Spatial Vision, 3, 179-197. doi: 10.1163/156856888X00122 WwW

Savelsbergh, G. J. P., Williams, A. M., Van Der Kamp, J., \& Ward, P. (2002). Visual search, anticipation and expertise in soccer goalkeepers. Journal of Sports Sciences, 20, 279-87. doi: 10.1080/026404102317284826 wWW

Simons, D., \& Chabris, C. F. (2010). The invisible gorilla: And other ways our intuitions deceive us. New York, NY: Crown.

Starkes, J. L., \& Ericsson, K. A. (2003). Expert performance in sports: Advances in research on sport expertise. Champaign, IL: Human Kinetics.

Terlecki, M. S., \& Newcombe, N. S. (2005). How important is the digital divide? The relation of computer and videogame usage to gender differences in mental rotation ability. Sex Roles, 53, 433-441. doi: 10.1007/s11199-005-6765-0

Trick, L. M., Jaspers-Fayer, F., \& Sethi, N. (2005). Multiple-object tracking in children: The "Catch the Spies" task. Cognitive Development, 20, 373-387. doi: 10.1016/j.cogdev.2005.05.009

Voss, M. W., Kramer, A. F., Basak, C., Prakash, R. S., \& Roberts, B. (2010). Are expert athletes 'expert 'in the cognitive laboratory? A meta-analytic review of cognition and sport expertise. Applied Cognitive Psychology, 24, 812-826. doi: 10.1002/ acp.1588

Voyer, D., Voyer, S., \& Bryden, M. P. (1995). Magnitude of sex differences in spatial abilities: A meta-analysis and consideration of critical variables. Psychological Bulletin, 117, 250-270. doi: 10.1037/0033-2909.117.2.250 [www]

Williams, A. M., \& Ford, P. R. (2008). Expertise and expert performance in sport. International Review of Sport and Exercise Psychology, 1, 4-18. doi: 10.1080/17509840701836867

RECEIVED 07.06.2016 | ACCEPTED 23.11.2016 